\title{
RURALIDAD, AGRICULTURA Y EXCLUSIÓN SOCIAL LOS EFECTOS DE LA DESIGUALDAD TERRITORIAL
}

\section{Luis Camarero y Julio A. del Pino}

\begin{abstract}
Resumen
Habitualmente se relaciona el atraso económico con la exclusión social de las áreas rurales. El texto aborda de manera independiente el efecto que tiene el hábitat - rural o urbano-dentro de los factores que inciden en la vulnerabilidad. Se analiza el indicador AROPE - riesgo de pobreza y exclusión-mediante un modelo de regresión logística que incluye el efecto de los factores sociodemográficos, estructuras de hogar y vinculación agraria- y la interacción con el hábitat, considerado como variable moderadora. Los resultados muestran que el medio rural reduce las diferencias en vulnerabilidad y proporciona un entorno menos desigual entre sus habitantes, debido a la internalización que hacen las estructuras de hogar de la posición periférica de las áreas rurales en el acceso a los servicios del bienestar.
\end{abstract}

Palabras clave: desagrarización, exclusión social, hábitat rural, bienestar.

\section{Abstract}

Rurality, agriculture and social exclusion. The territorial inequality effects

In general, social exclusion in rural areas is attributed to economic backwardness. The paper examines the rural-urban habitat contribution over the factors that produce vulnerability. The AROPE index - risk of poverty and exclusion - is analyzed by a logistic regression model that considers - socio-demographic household structures and agrarian bonding- as factors and their interaction with the habitat as a moderator variable. The findings show, by one hand, rural habitat reduces the vulnerability differences producing a less unequal environment among inhabitants. By another hand, it implies the internalization inside the households of the inequalities in the access of the rural population to the welfare services.

Keywords: de-agrarianization, social exclusion, rural habitat, welfare.

\footnotetext{
Luis Camarero: Doctor en Ciencias Políticas y Sociología. Catedrático de Sociología. Director del Departamento de Teoría, Metodología y Cambio Social, Universidad Nacional de Educación a Distancia (UNED), España. ORCID iD: 0000-0001-6665-2069

Email: Icamarero@poli.uned.es

Julio A. del Pino: Doctor en Sociología. Profesor contratado doctor en el Departamento de Teoría, Metodología y Cambio Social, UNED, España.

ORCID iD: 0000-0002-2737-2950

Email: jadelpino@poli.uned.es
} 


\section{Introducción}

La desagrarización, entendida como la reducción de la primacía social y económica que venía teniendo la actividad agraria en la vida de los pueblos, ha supuesto la diferenciación entre agricultura y ruralidad. Estos términos, aunque siguen empleándose como sinónimos, remiten a conceptos distintos. El hábitat rural ha reducido progresivamente su dependencia respecto de las economías agrarias, mientras ha ido diversificando sus actividades de subsistencia. En este proceso de cambio de actividades, se ha intensificado la inserción creciente de los habitantes rurales en mercados de trabajo extralocales a través del commuting o desplazamientos diarios desde sus lugares de residencia hasta los centros urbanos laborales. Entre tanto, la agricultura se ha industrializado y aumentado su productividad mediante la incorporación de capital y tecnología. Hay más agricultura y menos agricultores.

La desagrarización ha estado detrás del debate sobre la desigualdad social en el campo. La transformación que implica la denominada revolución verde en importantes regiones rurales produce un impacto grande en las sociedades campesinas y en las pequeñas explotaciones familiares que progresivamente van situándose en la periferia económica y social. La desvinculación agraria ha sido el principal argumento para interpretar el crecimiento de la exclusión social en buena parte de las regiones rurales. La explicación de la pobreza rural se ha centrado en la falta de oportunidades que produce el vacío agrario en el seno de las comunidades rurales. Rigg (2006) interpreta la desagrarización como una transición hacia la pobreza, mientras que para Grammont (2010) es un motor de precarización de la vida rural. En muchas regiones ni la emergencia de actividades no agrarias - turismo, servicios ambientales, artesanías-, ni tampoco el recurso de la emigración han sido suficientes para neutralizar el hueco que dejan los modos de vida agraria. Paradójicamente, se ha venido constatando un aumento del PIB agrario que, sorprendentemente, es compatible con el aumento de la pobreza de los hogares rurales (Graziano et al., 2008). En líneas generales, la modernización agraria ha alterado las fuentes de subsistencia rural, mientras la agricultura industrializada ha mantenido las condiciones de temporalidad, irregularidad y precariedad de la mano de obra agraria. Podadera et al. (2020) encuentran para el conjunto de Europa una relación especialmente elevada entre las tasas de población agraria y el nivel de exclusión social, mientras que en otras re- 
giones se viene indicando el proceso de desvinculación progresiva de la agricultura familiar (Craviotti y Gras, 2006) y su apartamiento, hasta el punto de la aparición de políticas específicas para la inclusión social de la agricultura familiar (Villarreal, 2018).

En este contexto, se ha venido considerando la cuestión rural asociada al atraso y a la falta de desarrollo. Sin embargo, la persistencia del declive rural, aun después de varias décadas de políticas de desarrollo rural, ha puesto en evidencia la importancia de otros factores menos estructurales y económicos. Así lo reconoce explícitamente la Unión Europea en la denominada Declaración de Cork 2.0 (Unión Europea, 2016). Los mecanismos explicativos acerca de la brecha rural han girado en torno a las lecturas del desarrollo regional. En este sentido, se enfatiza la idea que considera que la situación de las áreas rurales no es producto de la falta de desarrollo, sino del propio proceso de desarrollo. El actual modelo de acumulación se soporta en las economías de escala que funcionan mediante la concentración espacial de recursos, capital, mano de obra, consumidores y conocimiento. La posición periférica de las áreas rurales no es una circunstancia natural que vendría determinada por supuestas oportunidades limitadas, sino una consecuencia de un modelo de desarrollo que traslada la desigualdad económica — alimentada por la acumulación - a través de la desigualdad política territorializada.

El enfoque del "atraso rural" oculta la cuestión de la desigual atención política a los territorios rurales. En líneas generales, los derechos de ciudadanía y acceso a las condiciones del Estado de bienestar presentan una profunda brecha en función del tipo de hábitat de residencia. Esta desigualdad en el acceso a los recursos de bienestar, y no tanto el hecho de haber visto reducida su participación en la actividad agraria, es la que explicaría las diferencias en las condiciones de vida entre las áreas rurales y las urbanas (Camarero y Oliva, 2019). Dado el impacto cada vez más pequeño de la agricultura en la vida económica y social del hábitat rural, resulta plausible que las desigualdades persistentes en el territorio se nutran de las diferencias que, de manera creciente, encontramos en el acceso a los servicios públicos, tanto por la vía de la ausencia de recursos institucionales en los pueblos como por la dificultad de acceso a estos, algo que está mediado por la creciente importancia de la movilidad y la conectividad en el hábitat rural.

Este artículo pretende avanzar en la cuestión de las desigualdades sociales a través del indicador de riesgo de exclusión social. El análisis se dirige a determinar el impacto que tiene sobre las condiciones de vida el hecho de vivir en un área rural o en un área urbana, en contraste con el impacto que tiene la dedicación agraria.

Nuestra hipótesis huye de la idea habitualmente admitida de que la ruralidad es causa de exclusión y que confunde la asociación —entre desigualdad y ruralidad - con la explicación. En nuestro análisis vamos a considerar la 
variable hábitat como variable mediadora ${ }^{1}$ en la relación que se establece entre condición socioeconómica y exclusión social. Con este propósito, vamos a centrarnos no únicamente en los efectos de la variable, sino también en los efectos de interacción que tiene el hábitat con los factores de desigualdad. Nuestra sospecha es que las áreas rurales modifican la relación que se establece entre condición socioeconómica y exclusión. Los estudios sobre movilidad y desigualdades de género han alertado de este efecto. Por ejemplo, las desigualdades que produce la movilidad se hacen más intensas en las áreas rurales por el fuerte sobreenvejecimiento que existe en ellas (Walsh et al., 2017). Las desigualdades de género se amplifican en las áreas rurales por las mayores demandas de movilidad para la inserción en mercados laborales (Noack, 2010), por la mayor carga de dependencia y por la menor oferta de trabajo cualificado (Camarero et al., 2016).

En definitiva, podemos considerar que el hábitat rural no es fuente de desigualdades, sino que, en comparación con las áreas urbanas, las desigualdades resultan en él más intensas. Esa es nuestra idea: medir la intensificación que produce el hecho de ser residente rural en términos de exclusión social.

Para comprobar nuestra hipótesis realizamos un contraste mediante la técnica de regresión logística, para determinar el valor del riesgo de exclusión en cuanto variable dependiente en función de un conjunto de siete variables independientes que recorren dimensiones referidas tanto a los sujetos como al grupo doméstico. El modelo se contrasta en dos fases: una primera sin efectos de interacción y una segunda en la que se incluye la interacción que existe entre el hábitat y las distintas variables independientes. A partir de la existencia de interacción y de su dirección es posible determinar el efecto que tiene el hábitat como moderador - léase amplificador o reductor- de los procesos de desigualdad. Para una descripción más detallada del análisis de regresión logística y la consideración de interacción entre variables categóricas véase Jaccard (2001).

En esta investigación el hábitat rural se ha delimitado según el criterio de grado de urbanización que define Eurostat (Unión Europea, 2019) y que está vinculado a la densidad demográfica. El territorio se divide en celdas de un kilómetro cuadrado y se consideran rurales aquellas que tienen una población inferior a trescientos habitantes, siempre y cuando junto con las contiguas no superen un total de cinco mil habitantes. Los municipios en los que más de la mitad de la población reside en celdas rurales se denominan "zonas escasamente pobladas" o rurales y, en caso contrario, urbanas. En total, según esta clasificación un $26,1 \%$ de la población de España reside en un hábitat rural.

1 En términos estadísticos, se denomina moderadora porque su efecto puede modificar la relación entre las variables dependiente e independientes. 


\section{La tendencia del riesgo de exclusión social}

El tradicional término pobreza con el que se resumían las diferencias socioeconómicas ha sid sustituido por la noción de exclusión social. Este giro conceptual busca superar el reduccionismo económico en el estudio de las desigualdades para considerar otras dimensiones, al margen de la reducción de ingresos, como son el alejamiento del mercado de trabajo, el descenso en la participación en la vida social y, en consecuencia, una reducción y progresiva pérdida de derechos sociales e incluso cívicos (Laparra, 2001).

La Unión Europea, con la puesta en marcha del Tratado de Ámsterdam en 1999, comenzó a desarrollar una política activa en favor de la inclusión social, que se consolidó de forma específica en la denominada Estrategia de Lisboa (2000). Con ese propósito se diseñó un sistema de indicadores de medición y seguimiento de las condiciones y desigualdades socioeconómicas que, mediante la Encuesta de Ingresos y Calidad de Vida (Eurostat), recoge información de forma homogénea y comparable para los países miembros.

A partir de dicha encuesta se elabora el indicador de riesgo de exclusión social, denominado AROPE. ${ }^{2}$ Este indicador se basa en tres índices que recogen distintas dimensiones socioeconómicas:

- Carencia material: hogares que presentan al menos cuatro carencias dentro de un conjunto de nueve ítems. ${ }^{3}$

- Riesgo de pobreza: hogares que se sitúan por unidad de consumo con ingresos inferiores al $60 \%$ de la mediana.

- Hogares con muy baja intensidad de trabajo: personas de 0 a 59 años que viven en hogares en los que sus miembros en edad de trabajar lo hicieron menos de un $20 \%$ de su potencial total de trabajo, lo que se mide como la relación entre el número de meses trabajados durante el año por todos los miembros del hogar y el número de meses que dichos miembros podrían haber trabajado.

2 AROPE es el acrónimo de: at risk of poverty and/or exclusion.

3 1) No puede permitirse ir de vacaciones al menos una semana al año. 2) No puede permitirse una comida de carne, pollo o pescado al menos cada dos días. 3) No puede permitirse mantener la vivienda con una temperatura adecuada. 4) No tiene capacidad para afrontar gastos imprevistos (de 650 euros). 5) Ha tenido retrasos en el pago de gastos relacionados con la vivienda principal (hipoteca o alquiler, recibos de gas, comunidad...) o en compras a plazos en los últimos doce meses. 6) No puede permitirse disponer de un automóvil. 7) No puede permitirse disponer de teléfono. 8) No puede permitirse disponer de un televisor. 9) No puede permitirse disponer de una lavadora. 


\section{Cuadro 1. Distribución por hábitat de los índices que constituyen el AROPE. Porcentaje de población en cada hábitat}

\begin{tabular}{lcc} 
& Urbano & Rural \\
\hline Carencia material & $5,1 \%$ & $3,6 \%$ \\
\hline Riesgo de pobreza & $19,5 \%$ & $24,0 \%$ \\
\hline Baja intensidad de empleo & $10,4 \%$ & $12,3 \%$ \\
\hline AROPE & $\mathbf{2 4 , 1 \%}$ & $\mathbf{2 8 , 8 \%}$ \\
\hline
\end{tabular}

Fuente: Elaboración propia con base en ECV 2019, INE.

Un valor positivo en cualquiera de los tres indicadores implica un valor positivo en el AROPE. Los datos muestran que el indicador de rentas riesgo de pobreza- es el que tiene mayor incidencia en la tasa de riesgo de exclusión: el $83,3 \%$ de quienes están en riesgo presentan valores de renta insuficientes. Como puede observarse - Cuadro 1-, la exclusión social es importante y es mayor en las áreas rurales donde más de la cuarta parte de los habitantes se encuentra en esa situación. La única excepción en la desigual relación rural-urbano viene del índice de carencia material, para el cual las áreas rurales, en comparación con las urbanas, tienen ventaja. El motivo principal para ello es la concentración en áreas urbanas de las situaciones de infravivienda y de pobreza más extrema.

La evolución de los indicadores - Figura 1- muestra el importante efecto que ha tenido la crisis financiera de 2008. La Gran Recesión produjo un ascenso del indicador AROPE a cifras que en los años 2013 y 2014 llegaron a situar a más de la tercera parte de la población rural en riesgo de exclusión. La crisis supuso, además, un frenazo a la progresiva nivelación que venía observándose entre áreas urbanas y rurales por la destacable reducción de los valores de exclusión rural.

Si observamos la evolución en las áreas rurales en función de los componentes del indicador, podemos comprobar que el impacto de la crisis se ha concentrado especialmente en el empleo - Figura 2-. Así, el indicador de personas en hogares con baja intensidad de empleo ha visto multiplicar por cuatro sus valores hasta alcanzar, en los momentos más intensos de la crisis, casi a un $20 \%$ de la población. Como valor de referencia téngase en cuenta que una persona en situación de baja intensidad de empleo no alcanzaría a estar ocupada durante tres meses en un año. Paradójicamente, el indicador de rentas no se ha alterado con la misma intensidad. Este dato resulta clave y tiene dos lecturas. Por una parte, puede interpretarse en el sentido de que los sistemas de protección social han funcionado y las ayudas sociales han permitido mantener los niveles de renta. Por otra parte, hay que tener en cuenta el peso que tienen las pensiones en la renta de los hogares, especialmente en 
las áreas rurales sobreenvejecidas y que absorben en términos de bienestar global los efectos coyunturales.

Figura 1. Evolución del indicador AROPE según hábitat. 2004-2019

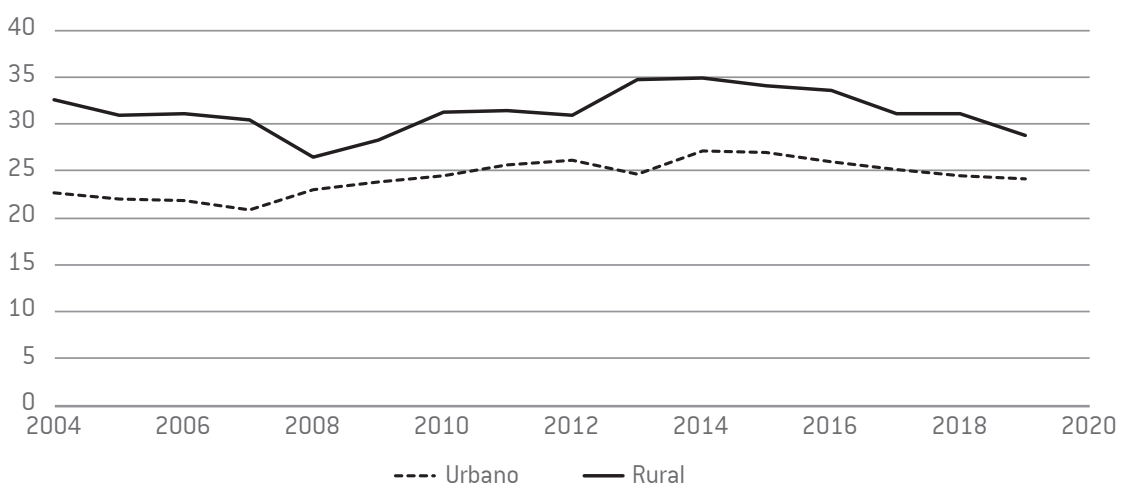

Fuente: Elaboración propia con base en ECV y Eurostat.

Figura 2. Evolución del AROPE y sus componentes para áreas rurales. 20042019

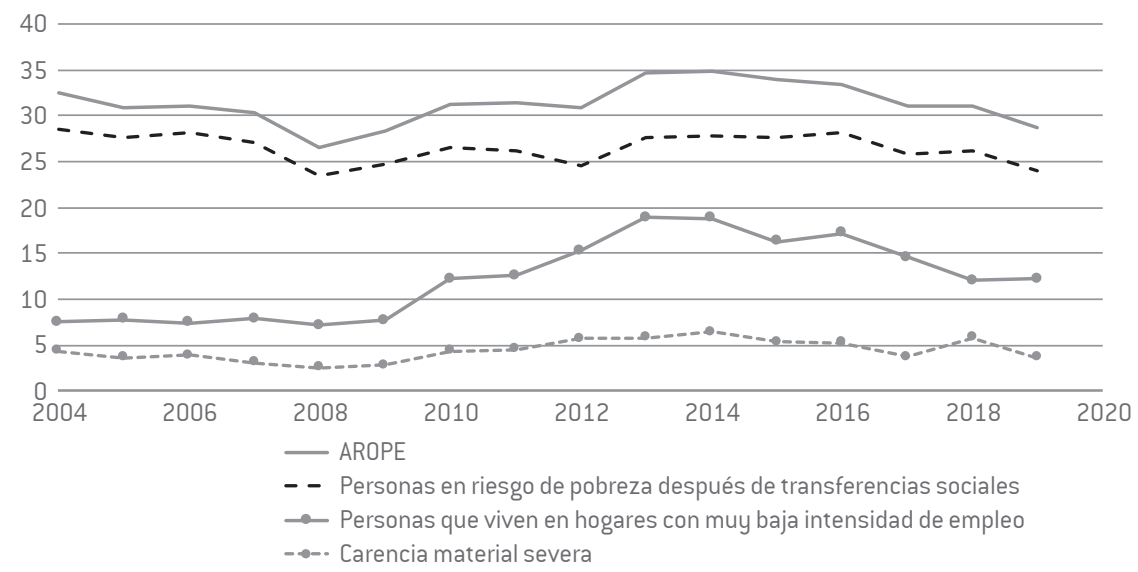

Fuente: Elaboración propia con base en ECV y Eurostat.

\section{Agricultura, ruralidad y estructuras de vulnerabilidad}

Nuestro modelo de análisis está influido por la factorización que Brugué et al. (2002) hacen de la exclusión social a través de los cambios que experimenta la estructura social, a la que refieren como fragmentación tridimensional de la sociedad. La diversificación étnica que han producido las grandes 
corrientes migratorias, los desequilibrios demográficos de una sociedad crecientemente envejecida y dependiente y la pluralidad de formas de convivencia diferentes a la familia nuclear sobre la que se basaban las estructuras de cuidados y de bienestar en los países que mantienen un Estado de bienestar rudimentario son tres líneas de cambio que alteran de forma conjunta el statu quo sobre el que se soporta el Estado de bienestar y transmiten nuevas fuentes de desigualdad social.

En nuestro análisis consideramos dos grupos de variables referidas al sujeto y al hogar o grupo doméstico de convivencia. En el Cuadro 2 se exponen las variables independientes utilizadas, así como su relación con la variable dependiente — riesgo de exclusión — y moderadora — hábitat—.

Nuestro diseño de investigación quiere aislar el posible efecto del hábitat en situaciones concretas. Buscamos separar qué parte del riesgo de exclusión se debe al efecto del hábitat y qué parte se debe a la probable acumulación de condiciones de exclusión en áreas rurales. En función de ello, construimos un modelo que incluye los efectos de interacción de las variables independientes con el hábitat y estudiamos su efecto.

Entre las variables independientes referidas al sujeto se consideran las características sociodemográficas de sexo y edad, ${ }^{4}$ así como las de nivel de estudios y país de nacimiento. La influencia del nivel de estudios y la condición de extranjero han sido continuamente destacadas en distintas investigaciones (Tsakloglou y Papadopoulos, 2002; Podadera et al., 2020) e influyen en las formas más precarias e inestables de inserción en los mercados de trabajo y acceso a distintas categorías profesionales. En este sentido, la diferencia nacional/extranjero es una variable determinante en los procesos de integración social. La importancia que tiene el proceso de etnofragmentación (Pedreño, 2005) o adscripción de las posiciones laborales al origen nacional es la base de los procesos de desregulación y precarización de los mercados de trabajo.

La composición del hogar nos permite situar a los grupos domésticos en función de las cargas familiares y acercarnos a las redes de apoyo y de cuidados familiares. Los analistas destacan el importante crecimiento de los hogares unipersonales y monoparentales, lo que ha sido relacionado con los procesos de exclusión (Tsakloglou y Papadopoulos, 2002). También se observa una redistribución de las formas de hogar en función del hábitat. Así, por ejemplo, en las áreas rurales el envejecimiento está produciendo un aumento de los hogares solitarios, mientras que en las áreas suburbanas y periurbanas se produce una mayor concentración de parejas jóvenes con hijos (Camarero y Pedreño, 2021). Precisamente, el diseño analítico empleado procura aislar la fuerte asociación que se produce entre ciertas categorías respecto al hábitat. 
Cuadro 2. Descripción de las variables independientes

\begin{tabular}{|c|c|c|c|c|}
\hline & Años & $\mathrm{N}$ & $\%$ rural & $\begin{array}{c}\text { \% riesgo de } \\
\text { exclusión }\end{array}$ \\
\hline \multirow{18}{*}{ Edad } & $0-4$ & 1422 & $24,1 \%$ & $31,3 \%$ \\
\hline & $5-9$ & 1931 & $24,9 \%$ & $27,6 \%$ \\
\hline & $10-14$ & 2232 & $24,5 \%$ & $30,3 \%$ \\
\hline & $15-19$ & 2238 & $25,1 \%$ & $32,7 \%$ \\
\hline & $20-24$ & 1935 & $26,2 \%$ & $33,9 \%$ \\
\hline & $25-29$ & 1752 & $24,5 \%$ & $28,3 \%$ \\
\hline & $30-34$ & 1769 & $25,9 \%$ & $28,2 \%$ \\
\hline & $35-39$ & 2262 & $26,2 \%$ & $26,0 \%$ \\
\hline & $40-44$ & 2976 & $25,1 \%$ & $21,9 \%$ \\
\hline & $45-49$ & 3169 & $24,1 \%$ & $26,7 \%$ \\
\hline & $50-54$ & 3166 & $25,0 \%$ & $27,8 \%$ \\
\hline & $55-59$ & 3263 & $27,4 \%$ & $30,0 \%$ \\
\hline & $60-64$ & 2892 & $25,5 \%$ & $21,5 \%$ \\
\hline & $65-69$ & 2369 & $26,6 \%$ & $14,8 \%$ \\
\hline & $70-74$ & 2120 & $27,8 \%$ & $14,1 \%$ \\
\hline & $75-79$ & 1770 & $29,9 \%$ & $15,0 \%$ \\
\hline & $80-84$ & 1230 & $32,5 \%$ & $20,5 \%$ \\
\hline & $85+$ & 1356 & $33,1 \%$ & $16,5 \%$ \\
\hline \multirow{2}{*}{ Sexo } & Hombre & 19368 & $26,9 \%$ & $24,6 \%$ \\
\hline & Mujer & 20484 & $25,4 \%$ & $26,0 \%$ \\
\hline \multirow{5}{*}{ Estudios } & Menos de primaria & 2769 & $40,6 \%$ & $36,7 \%$ \\
\hline & Primaria & 5118 & $32,7 \%$ & $28,7 \%$ \\
\hline & Secundaria grado 1 & 8423 & $31,8 \%$ & $31,7 \%$ \\
\hline & Secundaria grado 2 & 7541 & $23,2 \%$ & $23,7 \%$ \\
\hline & Superior & 9336 & $18,2 \%$ & $13,7 \%$ \\
\hline \multirow{2}{*}{ Lugar de nacimiento } & España & 29436 & $27,5 \%$ & $21,0 \%$ \\
\hline & Extranjero & 3757 & $20,0 \%$ & $46,3 \%$ \\
\hline
\end{tabular}




\begin{tabular}{|c|c|c|c|c|}
\hline & Años & N & $\%$ rural & $\begin{array}{l}\text { \% riesgo de } \\
\text { exclusión }\end{array}$ \\
\hline \multirow{9}{*}{ Composición del hogar } & Unipersonal 30-64 & 1732 & $23,8 \%$ & $33,1 \%$ \\
\hline & Unipersonal menor de 30 & 141 & $24,0 \%$ & $41,9 \%$ \\
\hline & Unipersonal mayor de 64 & 1900 & $27,2 \%$ & $16,6 \%$ \\
\hline & $\begin{array}{c}\text { Pareja, al menos uno es mayor de } \\
64, \text { sin hijos menores }\end{array}$ & 5424 & $28,8 \%$ & $19,1 \%$ \\
\hline & Pareja de menores de 65 sin hijos & 4336 & $24,1 \%$ & $21,6 \%$ \\
\hline & Otros hogares sin niños & 6271 & $28,7 \%$ & $21,9 \%$ \\
\hline & Monoparental & 1476 & $16,4 \%$ & $46,8 \%$ \\
\hline & Pareja con niños menores & 14040 & $25,4 \%$ & $25,2 \%$ \\
\hline & Otros hogares con niños & 4480 & $26,9 \%$ & $33,4 \%$ \\
\hline \multirow{4}{*}{$\begin{array}{l}\text { Vinculación del hogar } \\
\text { con la actividad }\end{array}$} & Activo no agrario & 30175 & $23,1 \%$ & $23,9 \%$ \\
\hline & Productor agrario & 723 & $76,5 \%$ & $22,0 \%$ \\
\hline & Asalariado agrario & 1164 & $53,7 \%$ & $45,7 \%$ \\
\hline & Inactivo & 7790 & $29,1 \%$ & $27,5 \%$ \\
\hline \multirow{2}{*}{ Hábitat } & Urbano & 28516 & & $24,1 \%$ \\
\hline & Rural & 11336 & & $28,8 \%$ \\
\hline Total & & 39852 & $26,1 \%$ & $25,3 \%$ \\
\hline
\end{tabular}

Fuente: Elaboración propia con base en ECV 2019.

El indicador de exclusión utilizado incluye de forma explícita la relación con el empleo, eje central de los procesos de exclusión social, y por ello no se recurre a los indicadores de ocupación. El propio AROPE nos informa al respecto. Sin embargo, en el caso que nos ocupa es fundamental considerar la vinculación agraria de los hogares. Como podemos comprobar - Cuadro 3- la desagrarización ha transformado de forma radical las formas de subsistencia de los hogares rurales.

En la actualidad, las dos terceras partes $(66,2 \%)$ de los hogares que guardan relación con la actividad agropecuaria son hogares en los que residen asalariados agrarios. La agricultura familiar en sentido estricto — en que al menos dos personas del hogar tienen ocupación agraria - roza, sin alcanzar, a un $6 \%$ de los hogares vinculados a la actividad agropecuaria. Dentro de la distinción clásica de la agricultura familiar como aquella en la que no existe vínculo salarial, encontramos la importancia que tienen los autónomos agrarios, que hemos incluido durante el análisis, junto a los productores familiares, en la misma categoría de productores agrarios. 


\section{Cuadro 3. Distribución de los hogares vinculados a la actividad agraria por} hábitat y tipo de hogar

\begin{tabular}{lcccccc}
\hline & \multicolumn{3}{c}{ Hogares en miles } & \multicolumn{2}{c}{$\begin{array}{c}\text { Hogares agrarios } \\
\text { por hábitat [\%] }\end{array}$} & $\begin{array}{c}\text { Hogares agrarios } \\
\text { por tipo [\%] }\end{array}$ \\
\cline { 2 - 7 } & Urbano & Rural & Total & Urbano & Rural & Total \\
\hline Familiar & 11,1 & 34,5 & 45,6 & $24,3 \%$ & $75,7 \%$ & $\mathbf{5 , 8 \%}$ \\
\hline Individual & 53,4 & 167,6 & 220,9 & $24,2 \%$ & $75,9 \%$ & $28,0 \%$ \\
\hline Asalariado & 236 & 285,1 & 521,1 & $45,3 \%$ & $\mathbf{5 4 , 7 \%}$ & $\mathbf{6 6 , 2 \%}$ \\
\hline $\begin{array}{l}\text { Total hogares } \\
\text { agrarios }\end{array}$ & 300,4 & 487,2 & 787,6 & $38,1 \%$ & $61,9 \%$ & $100 \%$ \\
\hline Total hogares & $13.815,79$ & $4.836,65$ & $18.652,44$ & & & \\
\hline $\begin{array}{l}\text { \% hogares } \\
\text { agrarios }\end{array}$ & $2,2 \%$ & $\mathbf{1 0 , 1 \%}$ & $4,2 \%$ & & & \\
\hline
\end{tabular}

Fuente: Elaboración propia con base en ECV 2019.

Los datos muestran el bajo impacto que tiene la actividad agraria en el medio rural. Únicamente uno de cada diez hogares tiene alguna vinculación total o parcial con el sector primario. Si bien se mantiene una relación importante entre agricultura en posiciones familiares y áreas rurales, es también destacable que uno de cada cuatro hogares agrarios familiares resida en áreas urbanas. En el caso de los asalariados, son rurales (54\%) pero también urbanos (46\%), dato que muestra el atractivo y las ventajas residenciales de las áreas urbanas que, por los beneficios de su localización, permiten con mayor facilidad desplazamientos a distintos lugares de trabajo y una mejora de las oportunidades laborales en contextos de estacionalidad. Los datos muestran una fotografía de una realidad agraria alejada de la agricultura familiar y, ciertamente, también respecto del propio hábitat rural y sus habitantes.

Los estudios han encontrado una relación inversa entre edad y exclusión (Tsakloglou y Papadopoulos, 2002). Es decir, hay más riesgo de exclusión en edades jóvenes que en edades mayores y esto es más acentuado en los países con un sistema del bienestar fuerte. El examen del riesgo de exclusión (AROPE) según la edad nos confirma este hecho - Figura 3-. El valor mínimo de exclusión se localiza en las edades más elevadas: especialmente entre los 65 y 80 años. Este dato enfatiza la importancia que tiene el sistema público de pensiones. Hay un segundo detalle también significativo. La distribución presenta una forma cíclica, con picos en las edades de 18 a 30 años y de 50 a 60, para situarse en mínimos entre los 30 y 50 años. El riesgo de exclusión se hace importante en los últimos años de la vida laboral, cuando la discontinuidad laboral se torna más prolongada, y también en los años jóvenes, 
cuando, por regla general, se produce una inserción precaria en los mercados laborales. Esto también hay que interpretarlo dentro del propio ciclo vital y de composición de los hogares. Así, el pico que alcanza el indicador de exclusión en jóvenes es efecto del crecimiento en términos de riesgo de la generación de sus padres, que empiezan a situarse en la cincuentena, con quienes conviven. A ello habría que sumar el mayor volumen de población de origen inmigrante en estas edades.

Asimismo, los datos admiten una interpretación longitudinal del cambio generacional. En cierta medida, la crisis económica de 2008 invirtió el sentido de progresión generacional del bienestar y es indicativo de ello que las pensiones de las personas retiradas superen de forma significativa el salario de las personas en edad joven, incluso en el contexto de los países mediterráneos, frente a los países del norte.

\section{Figura 3. Personas en hogares en riesgo de exclusión social por edad. Áreas rurales. 2019}

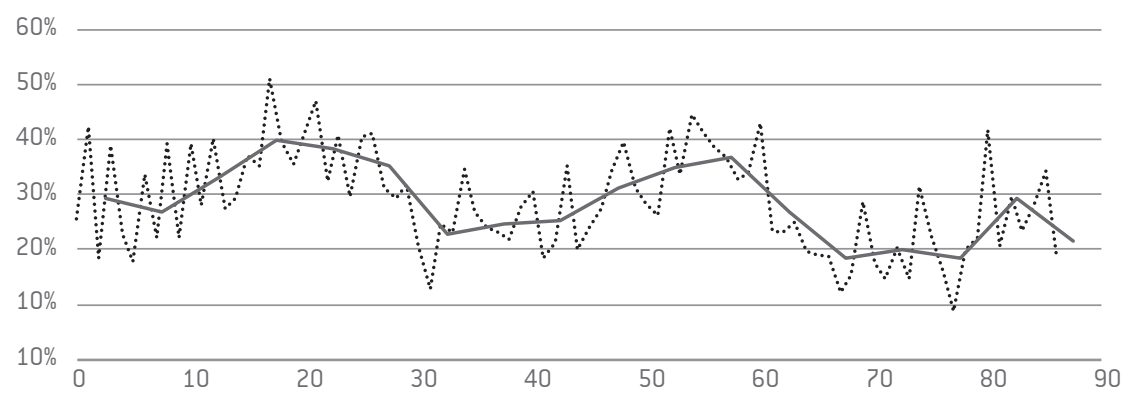

Nota: La línea punteada representa los datos brutos por edad en años, mientras que la línea sólida representa los datos agrupados en intervalos quinquenales y cada punto indica el valor medio del intervalo, con el objeto de hacer visible la tendencia.

Fuente: Elaboración propia con base en ECV 2019. 


\section{Factores de vulnerabilidad y efecto hábitat}

El Cuadro 4 recoge los coeficientes ${ }^{5}$ — $\beta$ y el odd-ratio $\mathrm{e}^{\beta}$ — de los dos modelos de regresión logística elaborados para explicar el riesgo de exclusión social. El modelo 1 contiene únicamente las variables independientes, de modo que permite observar la relación entre edad, sexo, hábitat, relación del hogar con la agricultura y estructura del hogar con la variable dependiente, que es el riesgo de pobreza y exclusión. Por su parte, el modelo 2 contiene los efectos de interacción de las variables independientes respecto de la variable hábitat, considerada moderadora de la relación. En este caso, la primera parte del cuadro nos permite observar los efectos de las variables una vez descontadas las diferencias estructurales entre el hábitat rural y urbano, mientras que la segunda parte mide, justamente, los efectos de la interacción entre cada variable y el hábitat. Todos los coeficientes en ambos modelos son significativos al menos para $\mathrm{p}<1 / 1.000$.

En la observación del modelo 1 -efectos directos - los valores de oddratio nos indican que el riesgo de exclusión disminuye ligeramente con la edad, cuestión que se había adelantado en la presentación de los datos. Es también mayor para las mujeres que para los hombres, lo que confirma lo que la literatura viene destacando. Pero si bien edad y sexo son variables significativas, es escasa su aportación en términos de exclusión respecto al resto.

La exclusión guarda una relación intensa y directa con el nivel de estudios. Podemos observar claramente que el incremento en el nivel de estudios reduce de manera proporcional el riesgo. Quienes no tienen estudios multi-

$5 \quad$ El lector que no esté especialmente familiarizado con los modelos de regresión logística puede considerar el coeficiente $\beta$ de manera similar a los coeficientes de regresión lineal. $\beta$ indicaría cuánto aumenta la variable dependiente ante una variación de la independiente, en el supuesto de que todas las demás variables independientes permanezcan fijas. La diferencia estriba en que la variable dependiente es el logaritmo del odd, que es la razón entre la probabilidad de que ocurra el suceso sobre la probabilidad de que no ocurra. Si bien $\beta$ nos informa de la intensidad y dirección de la relación, suele utilizarse $\mathrm{e}^{\beta}$ —odd-ratio - para realizar una lectura en términos de relación. El odd-ratio nos indica cuántas veces es más —o menos - probable que el suceso se produzca en relación con la categoría de referencia. En el Cuadro 3 para cada variable se han indicado las categorías de referencia entre paréntesis. Para la interpretación del cuadro, basta con fijarse en la odd-ratio $\left(\mathrm{e}^{\beta)}\right)$ de una de las categorías de una de las variables independientes del modelo, para conocer cómo influye y en qué sentido en la variable dependiente (riesgo de pobreza y exclusión), teniendo como referencia una de las categorías de la variable considerada. Por ejemplo, en el modelo 1, la odd-ratio de la categoría hombre es 0,909. Eso quiere decir que la probabilidad de entrar en riesgo de pobreza y exclusión en los varones es el 90,09\% de la probabilidad que tienen las mujeres. Aunque significativa, esta diferencia es muy pequeña y señala una mayor probabilidad de entrar en riesgo de pobreza de las mujeres que de los varones (de cada 100 mujeres que entraran, lo harían 90 varones), manteniendo todas las demás condiciones observadas inalteradas (a igual país de nacimiento, estudios, hábitat, relación con la agricultura y estructura de hogar). En el caso de los extranjeros, observamos una odd-ratio de 3,124 respecto de la categoría referente (nacidos en España). En este caso, ser extranjero eleva la probabilidad de entrar en riesgo de pobreza nada menos que un $312,4 \%$ respecto a ser español. Por cada español en riesgo de pobreza, hay más de 3 extranjeros en esa situación. 
plican por algo más de seis su riesgo de exclusión sobre quienes disponen de un título universitario. También, como era previsible, la exclusión guarda relación con el país de nacimiento, indicador que nos aproxima a procesos de segregación étnica. Quienes nacieron fuera de España multiplican por tres su riesgo de exclusión. Los estudios y la condición de inmigrante aparecen fuertemente asociados a la vulnerabilidad social. El capital formativo condiciona el acceso a trabajos cualificados y de mayor estabilidad, pero también la nacionalidad. Estudios y procedencia constituyen los principales factores de desigualdad.

La estructura por hogar señala que los hogares monoparentales concentran el mayor riesgo, así como también los hogares unipersonales de población joven y activa. Obsérvese que la categoría de referencia son los hogares unipersonales de entre 30 y 65 años que tienen coeficiente -odd-ratioigual a la unidad, valor que es claramente superior al que alcanzan los hogares compuestos por varias personas y generaciones. Estos datos nos confirman que las redes familiares son un claro protector frente a la exclusión.

Los datos muestran que el riesgo de exclusión es más intenso en áreas rurales. El hecho de residir en un área rural supone incrementar en un 20\% la probabilidad de exclusión sobre los habitantes urbanos. Es un valor relevante. Sin embargo, los hogares de los productores agrarios - categoría que puede ser interpretada como familia agraria - no aumentan su riesgo respecto al que tiene el conjunto de hogares activos. Como es esperable, es mayor y destacable el odd-ratio en asalariados agrarios y, evidentemente, en el conjunto de hogares inactivos, en cuyo caso el riesgo se hace tres veces mayor respecto al conjunto de hogares activos.

En líneas generales, los hallazgos no producen sorpresas: personas que viven solas y con menores a cargo, bajos estudios, inactivos e inmigrantes componen el perfil de la exclusión, que se acentúa algo más entre mujeres y jóvenes. A ello se añade la condición de rural, pero no necesariamente la condición de agricultor. Esta es la principal novedad. Incluso podemos destacar que, aunque sea muy ligeramente, los hogares de productores agrarios - empresarios y autónomos - reducen el riesgo sobre el conjunto (tienen un odd próximo pero inferior a la unidad). Ahora bien, la posición en la actividad agraria sí que condiciona la exclusión: los asalariados tienen una penalización de riesgo de $40 \%$ sobre quienes están en posiciones familiares $(1,361 / 0,966=1,409)$.

El segundo modelo explora de forma concreta el efecto del hábitat a través de su interacción con cada una de las categorías de las variables independientes. El primer hallazgo tiene que ver con su constatación. El análisis del producto de variables es significativo en todos los casos, al menos con una probabilidad de rechazo inferior a 1/1000. La interacción existe. Esto quiere decir que el riesgo de exclusión aumenta o disminuye para cada categoría en función del hábitat, tal y como habíamos sospechado. El sexo, los estudios o 
la composición familiar tienen efectos distintivos en áreas rurales y urbanas. No es lo mismo ser, por ejemplo, mujer o extranjero en un lugar urbano o en uno rural.

El análisis nos permite precisar aún más. Así, podemos observar el efecto de las variables descontando su interacción - interferencia- con el hábitat (los coeficientes con los efectos de interacción de las variables con el hábitat están situados en las últimas líneas del Cuadro 4). En líneas generales, se observa la misma estructura que habíamos detallado. El riesgo es inverso a la edad y ligeramente mayor para las mujeres, pero ahora el efecto directo del hábitat es nimio, incluso podría interpretarse que reduce la probabilidad de exclusión. Obsérvese que el odd-ratio rural es de 0,943, muy próximo a la unidad, que es el valor urbano de referencia. Los estudios y el país de nacimiento mantienen la dirección de sus efectos, se penalizan los niveles bajos de estudio y la condición de extranjero, pero esta penalización se hace más intensa si descontamos el efecto hábitat. Ello quiere decir que el hábitat estaba moderando dicha relación. ¿Cuál es el sentido de la moderación? Para ambas variables el medio rural reduce el efecto, es decir, en áreas rurales el nivel de estudios produce menores diferencias que en áreas urbanas. 


\section{Cuadro 4. Riesgo de pobreza y exclusión social. Modelos de regresión logística}

\begin{tabular}{|c|c|c|c|c|}
\hline & \multicolumn{2}{|c|}{ Modelo 1} & \multicolumn{2}{|c|}{ Modelo 2} \\
\hline & B & $\mathrm{e} \beta$ & B & $e ß$ \\
\hline Edad &,- 111 & ,895 &,- 116 & ,890 \\
\hline \multicolumn{5}{|l|}{ Sexo (mujer) } \\
\hline Hombre &,- 095 & ,909 &,- 083 & ,921 \\
\hline \multicolumn{5}{|l|}{ País de nacimiento [España] } \\
\hline Extranjero & 1,139 & 3,124 & 1,208 & 3,345 \\
\hline \multicolumn{5}{|l|}{ Estudios (superiores) } \\
\hline Inferiores a básicos & 1,918 & 6,810 & 2,143 & 8,529 \\
\hline Básicos & 1,358 & 3,887 & 1,420 & 4,136 \\
\hline Medios grado 1 & 1,146 & 3,145 & 1,203 & 3,330 \\
\hline Medios grado 2 & ,520 & 1,682 &, 573 & $1,7>4$ \\
\hline \multicolumn{5}{|l|}{ Hábitat (urbano) } \\
\hline Rural & ,195 & 1,216 &,- 059 & ,943 \\
\hline \multicolumn{5}{|l|}{ Hogar agrario (activo no agrario) } \\
\hline Productor agrario &,- 035 &, 966 & $-1,495$ & ,224 \\
\hline Asalariado agrario & ,308 & 1,361 & 021 & 1,021 \\
\hline Inactivo & 1,109 & 3,031 & 1,056 & 2,874 \\
\hline \multicolumn{5}{|l|}{ Estructura de hogar (unipersonal de 30 a 65) } \\
\hline Unipersonal menor de 30 & ,234 & 1,264 & ,064 & 1,066 \\
\hline Unipersonal mayor de 64 & $-1,797$ & ,166 & $-1,7>6$ & ,169 \\
\hline $\begin{array}{l}\text { Pareja, al menos uno es mayor de } 64 \text {, sin } \\
\text { hijos menores }\end{array}$ & $-1,415$ & ,243 & $-1,518$ & ,219 \\
\hline Pareja de menores de 65 sin hijos &,- 700 & ,497 &,- 803 & ,448 \\
\hline Otros hogares sin niños &,- 747 &, 474 &,- 782 & ,458 \\
\hline Monoparental & ,306 & 1,358 & ,239 & 1,270 \\
\hline Pareja con niños &,- 546 &, 579 &,- 629 &, 533 \\
\hline Otros hogares con niños &,- 503 &, 605 &,- 634 &, 530 \\
\hline \multicolumn{5}{|l|}{ Edad por hábitat (urbano) } \\
\hline Edad: rural & & & ,021 & 1,022 \\
\hline \multicolumn{5}{|l|}{ Sexo por hábitat (mujeres: urbano] } \\
\hline Hombres: rural & & &,- 022 & ,978 \\
\hline
\end{tabular}




\begin{tabular}{|c|c|c|c|c|}
\hline & \multicolumn{2}{|c|}{ Modelo 1} & \multicolumn{2}{|c|}{ Modelo 2} \\
\hline & B & $e ß$ & B & $e ß$ \\
\hline \multicolumn{5}{|l|}{$\begin{array}{l}\text { País de nacimiento por hábitat (España: } \\
\text { urbano) }\end{array}$} \\
\hline Extranjero: rural & & &,- 304 & ,738 \\
\hline \multicolumn{5}{|l|}{ Estudio por hábitat (superiores: urbano) } \\
\hline Inferiores a básicos: rural & & &,- 709 & ,492 \\
\hline Básicos: rural & & &,- 306 & ,737 \\
\hline Medios grado I: rural & & &,- 271 & ,763 \\
\hline Medios grado II: rural & & &,- 253 & 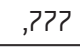 \\
\hline \multicolumn{5}{|l|}{$\begin{array}{l}\text { Hábitat por tipo de hogar agrario (hogar activo } \\
\text { no agrario: urbano] }\end{array}$} \\
\hline Hogar de productores agrarios: rural & & & 1,705 & 5,502 \\
\hline Hogar de asalariados agrarios: rural & & &, 561 & 1,752 \\
\hline Hogar de inactivos: rural & & & ,223 & 1,249 \\
\hline \multicolumn{5}{|l|}{$\begin{array}{l}\text { Estructura de hogar por hábitat (unipersonal } \\
\text { menor de 65: urbano) }\end{array}$} \\
\hline Unipersonal menor de 30: rural & & & ,639 & 1,895 \\
\hline Unipersonal mayor de 64: rural & & &,- 066 & ,936 \\
\hline $\begin{array}{l}\text { Pareja, al menos uno es mayor de } 64 \text {, sin } \\
\text { hijos menores: rural }\end{array}$ & & & ,316 & 1,371 \\
\hline Pareja de menores de 65 sin hijos: rural & & & ,386 & 1,470 \\
\hline Otros hogares sin niños: rural & & &, 127 & 1,136 \\
\hline Monoparental: rural & & & ,224 & 1,251 \\
\hline Pareja con niños: rural & & & ,318 & 1,375 \\
\hline Otros hogares con niños: rural & & &, 448 & 1,565 \\
\hline Constante &,- 515 & ,597 &,- 442 & ,643 \\
\hline
\end{tabular}

Significación menor de 1/10.000. En negrita significación menor de 1/1.000.

Modelo 1: $R_{\text {Cox-Snell }}^{2}=0,116 ; R^{2}$ Nagelkerke $=0,173$

Modelo 2: $R_{\text {Cox-Snell }}^{2}=0,118 ; R^{2}$ Nagelkerke $=0,177$

Fuente: Elaboración propia.

Para comprender mejor la incidencia de la interacción calculamos, a partir de la función logit resultante de la regresión logística realizada, las probabilidades pronosticadas para el cruce de variables respecto del hábitat, manteniendo el resto de las variables constantes según las categorías utilizadas como referencia. Los resultados aparecen en el Cuadro 5. Estas probabi- 
lidades debemos interpretarlas como las probabilidades de exclusión social para el conjunto de la categoría. Así, por ejemplo, el riesgo de exclusión de un extranjero que reside en áreas rurales es de 0,599 (59,9\%), valor que es inferior al riesgo que tiene un extranjero que reside en áreas urbanas $(68,3 \%)$ - con la misma edad, el mismo sexo, el mismo nivel de estudio, etcéteraPara valorar las diferencias nos valemos de la ratio entre las probabilidades por hábitat. Cuando es inferior a 1, las ventajas son rurales. Descontando la interacción, encontramos que la desigualdad es menor en las áreas rurales y especialmente apreciable para el grupo de nacidos fuera de España, en comparación con las áreas urbanas.

\section{Cuadro 5. Probabilidades estimadas de exclusión. Lugar de nacimiento por hábitat}

\begin{tabular}{lccc} 
& Urbano & Rural & Ratio rural/urbano \\
\hline Español & 0,391 & 0,377 & 0,964 \\
\hline Extranjero & 0,683 & 0,599 & 0,877 \\
\hline
\end{tabular}

Probabilidades ajustadas con interacción manteniendo el resto de las variables constantes.

Fuente: Elaboración propia.

Los extranjeros en áreas rurales reducen sus diferencias respecto a los habitantes urbanos. El medio rural reduce el peso que tienen estas diferencias individuales y, en definitiva, iguala a sus habitantes en cuanto a probabilidades de exclusión, mientras que en las áreas urbanas estas diferencias se acrecientan.

Si examinamos la variable que mayor incidencia ha mostrado en el riesgo de exclusión, el nivel de estudios, observamos - Cuadro 6- que las distancias entre los extremos - sin estudios y universitarios - son menos discriminantes en áreas rurales. La distancia entre universitarios y sin estudios en áreas urbanas es $0,846-0,391=0,455$ y en áreas rurales $0,718-0,377=0,341$. La mejora se produce principalmente en las categorías sin estudios. Es decir, para bajos niveles de estudios el medio rural resulta menos discriminante.

Ahora bien, respecto a la estructura de los hogares el efecto que juega el hábitat es el contrario. En los efectos directos del modelo 2 la variable mantiene sus diferencias - recordemos, monoparentales y hogares unipersonales frente a parejas y familias nucleares-. Sin embargo, los coeficientes - odd-ratio - del producto de variables invierten sus valores, que ahora son generalmente superiores a la unidad, indicando peor situación en cuanto a riesgo. Desde la perspectiva de las estructuras de hogar, el medio rural muestra diferencias relevantes respecto a las áreas urbanas. La lectura de las probabilidades estimadas nos muestra que las formas familiares son las más 
penalizadas para las áreas rurales, a excepción de los jóvenes solitarios, las parejas y quienes tienen niños.

Cuadro 6. Probabilidades estimadas de exclusión. Nivel de estudios por hábitat

\begin{tabular}{llll} 
& Urbano & Rural & Ratio rural/urbano \\
\hline Inferiores a básicos & 0,846 & 0,718 & 0,849 \\
\hline Básicos & 0,727 & 0,649 & 0,893 \\
\hline Medios grado 1 & 0,682 & 0,606 & 0,889 \\
\hline Medios grado 2 & 0,533 & 0,455 & 0,854 \\
\hline Superiores & 0,391 & 0,377 & 0,964 \\
\hline
\end{tabular}

Probabilidades ajustadas con interacción manteniendo el resto de las variables constantes.

Fuente: Elaboración propia.

Cuadro 7. Probabilidades estimadas de exclusión. Nivel de estudios por hábitat

\begin{tabular}{llcc}
\hline & Urbano & Rural & $\begin{array}{c}\text { Ratio rural/ } \\
\text { urbano }\end{array}$ \\
\hline Unipersonal 30-64 & 0,391 & 0,377 & 0,964 \\
\hline Unipersonal menor de 30 & 0,407 & 0,550 & 1,351 \\
\hline Unipersonal mayor de 64 & 0,098 & 0,088 & 0,898 \\
\hline Pareja, al menos uno mayor de 64, sin hijos menores & 0,123 & 0,154 & 1,252 \\
\hline Pareja, menores de 65, sin hijos & 0,224 & 0,285 & 1,272 \\
\hline Otros hogares sin niños & 0,227 & 0,239 & 1,053 \\
\hline Monoparental & 0,449 & 0,491 & 1,094 \\
\hline Pareja con niños & 0,255 & 0,307 & 1,204 \\
\hline Otros hogares con niños & 0,254 & 0,335 & 1,319 \\
\hline Probs
\end{tabular}

Probabilidades ajustadas con interacción manteniendo el resto de las variables constantes.

Fuente: Elaboración propia.

Lo que nos dicen los datos es que el hábitat rural nos iguala individualmente - estudios u origen pierden peso-, pero las formas familiares - con la excepción de los jóvenes solitarios- resultan más perjudicadas respecto al grupo de hogares solitarios. 


\section{Cuadro 8. Probabilidades estimadas de exclusión. Tipo de hogar agrario por hábitat}

\begin{tabular}{lccc} 
& Urbano & Rural & Ratio rural/urbano \\
\hline Activo no agrario & 0,391 & 0,377 & 0,964 \\
\hline Productor agrario & 0,126 & 0,428 & 3,397 \\
\hline Asalariado agrario & 0,396 & 0,520 & 1,313 \\
\hline Inactivo & 0,649 & 0,685 & 1,055 \\
\hline
\end{tabular}

Probabilidades ajustadas con interacción manteniendo el resto de las variables constantes.

Fuente: Elaboración propia.

La relación entre hábitat y ocupación de los hogares conduce a resultados relevantes. En líneas generales, atendiendo al odd-ratio, los hogares agrarios, descontando la interacción del hábitat, mejoran su situación, tienen $4,5(1 / 0,224=4,46)$ veces menor riesgo de exclusión que el conjunto de otros hogares de ocupados. El coeficiente de asalariados alcanza un valor casi idéntico a la unidad, es decir, no hay diferencias sustantivas respecto a la situación general de ocupación. Ahora bien, si consideramos el efecto de interacción con el hábitat, se acentúan las diferencias (Cuadro 8). Tanto para productores como para asalariados agrarios el riesgo de exclusión crece en áreas rurales. La categoría de agricultores familiares - productores agrarios- multiplica por más de tres el riesgo y alcanza valores de exclusión superiores a $42 \%$. Mientras que, como hemos observado, en los efectos directos la actividad agraria no guarda relación con la exclusión, sin embargo, el hecho de que sean agricultores familiares pero también asalariados que residen en áreas rurales amplifica su riesgo de exclusión. En el caso de la agricultura, no es la actividad la que está asociada a la exclusión, sino el entorno - el hábitat rural- el principal elemento de exclusión.

\section{Discusión}

El análisis realizado nos ha permitido introducirnos en la cuestión generalmente observada de la mayor vulnerabilidad en la que se encuentra la población en el hábitat rural. Los análisis agraristas determinaban que dicha situación era producto de la marginalidad en la que se encontraban las economías agrarias. El contexto de la nueva ruralidad desmonta dicha explicación. La actividad agraria no es dominante e incluso, en comparación con otras actividades productivas, difícilmente puede considerarse marginal.

Los datos nos han mostrado el efecto que tiene el hábitat en la reproducción de la desigualdad social. Hemos explorado qué diferencias produce dentro del conjunto de vectores que construyen las situaciones de vulnerabilidad. 
La lectura nos ha permitido señalar que, comparativamente respecto a las áreas urbanas, las áreas rurales son menos desiguales, es decir, neutralizan el efecto de los principales vectores. Si se observa más desigualdad es por el hecho de que los residentes presentan condiciones más elevadas para ello. Pero cuando neutralizamos las diferencias de composición, descubrimos que, al contrario, la distancia respecto al riesgo de exclusión se reduce. El medio rural modera las diferencias que niveles de estudio o procedencia nacional pueden tener, mientras que las áreas urbanas intensifican el efecto que tienen estas características.

Esta primera constatación es muy valiosa de cara al abordaje de las diferencias rural-urbano. Hay mayor desigualdad, pero porque se concentran con mayor intensidad perfiles asociados a un mayor riesgo. Las áreas rurales han venido experimentando una continua extracción de talento y de potencial en términos de capital social (Camarero y Pedreño, 2021). Los datos descriptivos muestran claramente las diferencias. Pero este proceso es también patente en el grupo de inmigrantes. Quienes tienen estudios se desplazan progresivamente hacia las ciudades, mientras entre los inmigrantes que llegan a las áreas rurales lo hacen en mayor proporción quienes tienen menor nivel educativo. Al filtrar el efecto de la interacción que supone el hábitat emerge la asociación de inmigración con nivel de estudios, situación que supone la acumulación de condiciones de vulnerabilidad, pero que no es atribuible a la condición de hábitat rural.

Neutralizado este efecto observamos, por el contrario, la capacidad de las áreas rurales para proporcionar un marco más igualitario. Estos datos recalcan la importancia y el efecto que tiene el modelo de desarrollo basado en la concentración (Rodríguez-Pose, 2018), que produce a la larga situación de exclusión en los lugares de emisión de trabajadores y de extracción de capacidad vital.

Pero nuestra hipótesis incluía la idea de que las desigualdades son producto de las diferencias que existen en el hábitat en cuanto a oferta y acceso al bienestar. En esta dirección podemos destacar la progresiva concentración de la fecundidad en las áreas periurbanas (Kulu y Boye, 2009) por la continua emigración de las parejas jóvenes hacia estos lugares. Son localidades donde la conexión con las oportunidades educativas y servicios asistenciales es menos onerosa en tiempo-coste y por ello resultan más atractivas. Las parejas jóvenes rurales terminan desplazándose, de forma que optimizan la relación en el espacio entre ofertas laborales y demandas asistenciales.

En este sentido podemos interpretar otro hallazgo relevante que nos han ofrecido los datos. Mientras las áreas rurales moderan el efecto de los factores de desigualdad, penalizan a los grupos familiares, especialmente a los grupos nucleares. Este hecho confirma la hipótesis de partida que señalaba la fuerte incidencia que cobran las desigualdades en el acceso al bienestar en función del hábitat. 
Es relevante que las diferencias y el impacto de la desigualdad aparezcan cuando consideramos las estructuras de hogar. Por una parte, nos habla de la mayor hostilidad que produce el hábitat respecto al desarrollo vital de los hogares, pero, por otra, también destaca el carácter resiliente que adquieren las estructuras familiares rurales y que ya habíamos advertido en estudios anteriores (Camarero y del Pino, 2014). El análisis realizado permite afirmar que para los perfiles de mayor exclusión la reducción del impacto que producen las áreas rurales puede explicarse por la colectivización de dichas diferencias en el interior de los grupos domésticos. Los grupos familiares que residen en las áreas rurales son fuertemente adaptativos y neutralizan, mediante las redes de apoyo y cuidados, el hándicap que supone la menor accesibilidad a los servicios de bienestar, pero también mediante las estrategias familiares de subsistencia consiguen absorber las condiciones de exclusión individuales. Por ejemplo, como ha señalado Pages (2011) para la Francia rural, mediante la extensión de la convivencia intergeneracional — hijos mayores con padres - o la vuelta de hijos al hogar doméstico para hacerse cargo de los padres.

Una última cuestión queda también abierta para la reflexión. Los datos han mostrado claramente la polaridad que establecen los hogares agrarios en función del hábitat. Ciertamente, el lugar de residencia segmenta la agricultura familiar en dos grupos: los agricultores residentes en áreas urbanas, que se sitúan en posiciones alejadísimas de la consideración de exclusión, mientras que la agricultura familiar ejercida por residentes rurales adquiere valores muy altos de riesgo de exclusión. No es la actividad agraria, sino la relación entre ruralidad y agricultura donde se concentra la desigualdad. Reconocemos así la dualidad que ya habían señalado Riella y Romero (2003) entre un modelo de desarrollo agrario - que en nuestro caso se hace progresivamente urbano- - y un modelo de desarrollo que expulsa población agraria e incrementa el riesgo de exclusión de los productores agrarios residentes en el hábitat rural.

En definitiva, el análisis destaca la importancia que tienen las diferencias entre el hábitat rural y el urbano, pero no en cuanto al atraso económico, sino en cuanto a la posición periférica del hábitat rural en el contexto del Estado de bienestar. Escribano et al. (2019) han señalado las dificultades que tiene el indicador AROPE para medir la exclusión social en entornos territoriales concretos. Estos autores han puesto de manifiesto la importancia que juegan las variables territoriales — distancia, dispersión y movilidad - en situar el riesgo en distintos territorios. Efectivamente, señalan la importancia que tiene la cohesión territorial, siendo el Estado del bienestar y sus políticas el mecanismo para reducir la fricción que producen la distancia, la dispersión y las carencias de movilidad que, como hemos visto, condicionan las oportunidades vitales de las áreas rurales. Las poblaciones de las áreas rurales han mostrado su capacidad adaptativa frente a la insolidaridad orgánica desplegada territorialmente. 


\section{Referencias bibliográficas}

Brugué, Q.; R. Gomá y J. Subirats (2002). De la pobreza a la exclusión social: nuevos retos para las políticas públicas. Revista Internacional de Sociología, 60(33), pp. 7-45.

Camarero, L.; F. Cruz y J. Oliva. (2016). Rural sustainability, inter-generational support and mobility. European Urban and Regional Studies, 23(4), pp. 734-749.

Camarero, L. y J. del Pino (2014). Cambios en las estructuras de los hogares Rurales. Formas de adaptación y resiliencia. Revista Internacional de Sociología, 72(2), pp. 377-401.

Camarero, L. y J. Oliva (2019). Thinking in rural gap: mobility and social inequalities. Palgrave Communications, 5(95), pp. 1-7.

Camarero, L. y A. Pedreño (2021). Globalización y territorios: despoblación, geografías de la periferia y ciudadanía. En: G. Ubasart-González y R. Gomà (eds.). Vidas en transición. (Re)construir la ciudadanía social. Madrid: Tecnos, pp. 124-144.

Craviotti, C. y C. Gras (2006). De desafiliaciones y desligamientos: trayectorias de productores familiares expulsados de la agricultura pampeana. Desarrollo Económico, 46(181), pp. 117-134.

Escribano, J.; J. Serrano y P. Martínez (2019). Análisis del riesgo de exclusión social en el medio rural. El índice Z como solución "lowcost" a la falta de indicadores sintéticos municipales. Cuadernos Geográficos, 58(3), pp. 103-124.

Grammont, H. (2010). Nueva ruralidad: ¿un concepto útil para repensar la relación campo-ciudad en América Latina? Ciudades, 85, pp. 2-20.

Graziano, J.; S. Gómez y R. Castañeda (2008). Boom agrícola y persistencia de la pobreza en América Latina. Revista Española de Estudios Agrosociales y Pesqueros, 218, pp. 17-44.

Jaccard, J. (2001). Interaction Effects in Logistic Regression. Thousand Oaks, CA: Sage Publications.

Kulu, H. y P. Boye (2009). High Fertility in City Suburbs: Compositional or Contextual Effects? European Journal of Population, 25, pp.157-174.

Laparra, M. (2001). Una perspectiva de conjunto sobre el espacio social de la exclusión. En: L. Moreno (coord.). Pobreza y exclusión: la "malla de seguridad” en España. Madrid: CSIC, pp. 53-78. 
Noack, E. (2010). Are Rural Women Mobility Deprived? - A Case Study from Scotland. Sociologia Ruralis, 51(1), pp. 79-97.

Pages, A. (2011). La Pauvreté en Milieu Rural. Tolouse: Presses universitaires du Midi.

Pedreño, A. (2005). Sociedades etnofragmentadas. En: A. Pedreño y M. Hernández (coords.). La condición inmigrante. Murcia: Universidad de Murcia, pp. 75-103.

Podadera, P.; A. Martín-Cambero; A. García y J. Lobato (2020). Pobreza y exclusión social en la Unión Europea. Revista de Economía Mundial, 56, pp. 143-164.

Riella, A. y J. Romero (2003). Nueva ruralidad y empleo no agrícola en Uruguay. En: M. Bendini y N. Steimbreger (eds). Territorios y organización social de la agricultura. Buenos Aires: GESA/Editor La Colmena, pp. 157-164.

Rigg, J. (2006). Land, Farming, Livelihoods, and poverty: Rethinking the links in the rural south. World Development, 34(1), pp. 180-202.

Rodríguez-Pose, A. (2018). The revenge of the places that don't matter (and what to do about it). Cambridge Journal of Regions, Economy and Society, 11(1), pp. 189-209.

Tsakloglou, P. y F. Papadopoulos (2002). Aggregate level and determining factors of social exclusion in twelve European countries. Journal of European Social Policy, 12(3), pp. 211-235.

Unión Europea (2016). Declaración de Cork 2.0. Una vida mejor en el medio rural. Luxemburgo: Oficina de Publicaciones de la Unión Europea.

Unión Europea: (2019) Methodological manual on territorial typologies-2018 edition. Luxemburgo: Oficina de Publicaciones de la Unión Europea.

Villarreal, F. (2018). La inclusión de la agricultura familiar: discusión de su uso en programas de desarrollo rural en Argentina. Mundo Agrario, 19(41), e091.

Walsh, K.; T. Scharf y N. Keating (2017). Social exclusion of older persons: a scoping review and conceptual framework. European Journal of Ageing, 14, pp. 81-98.

\section{Contribución de autoría}

Este trabajo fue realizado en partes iguales por Luis Camarero y Julio A. del Pino. 